\title{
A Comparative Analysis of Monte Carlo and Quasi-Monte Carlo Methods in Financial Derivative Pricing
}

\author{
S. M. Arif Hossen* and A B M Shahadat Hossain \\ Department of Applied Mathematics, University of Dhaka, Dhaka-1000, Bangladesh
}

(Received : 24 November 2019; Accepted : 24 February 2021)

\begin{abstract}
The main purpose of this dissertation is to study Monte Carlo (MC) and Quasi-Monte Carlo (QMC) methods for pricing financial derivatives. We estimate the Price of European as well as various path dependent options like Asian, Barrier and American options by using these methods. We also compute the numerical results by the above mentioned methods and compare them graphically as well with the help of the MATLAB Coding.
\end{abstract}

Keywords: Monte Carlo (MC) method, Quasi-Monte Carlo (QMC) method, European option, American option, Asian option, Barrier option, Pseudorandom number, Halton sequence, Sobol sequence, Black Scholes model.

\section{Introduction}

The Monte Carlo (MC) method and its deterministic version Quasi-Monte Carlo (QMC) method are popular numerical tools in pricing financial derivatives and risk management. Monte-Carlo method and Quasi Monte-Carlo method were both used in physics at the beginning, so most solutions were given by physicists. There had been a controversy about whether these solutions also applicable in finance and derivatives pricing. Corwin Joy $^{8}$ (Corwin, 1996) pointed out the disadvantage of Monte-Carlo and raised Quasi Monte-Carlo. They substituted random sequence with deterministic sequence which can improve convergent rate and have a deterministic error interval. ${ }^{5}$

The MC method simulates the underlying model using random numbers, and estimates the expectation of the random variable by sample averages. The QMC method attempts to do the estimation similar to the MC method with one crucial difference that it uses the so-called lowdiscrepancy sequences in simulation. These are specially designed number theoretic sequences for estimating integrals. For this reason, the QMC method is often called the deterministic version of the MC method. There are two advantages of using the QMC method: it provides deterministic convergence, as opposed to the stochastic convergence of MC method. Moreover, the QMC estimates converge to the true solution at the rate $O\left((\log N)^{d} / N\right)$, where $N$ is sample size and $d$ is the dimension of the problem whereas the MC convergence rate is $O\left(N^{-1 / 2}\right)$. The QMC method has some drawbacks: first, using one low discrepancy sequence in simulation gives a single estimate for the integral, and there is no practical way of assessing the error of this estimate. The second drawback is due to the deterioration of low discrepancy sequences in high dimensions. The third main shortcoming is due to the lack of practical bounds for integration error in QMC methods. Therefore, more practical error bounds are needed in QMC integration. ${ }^{5}$ Geometric Brownian motion is a standard model used in financial mathematics, and the MC method is often used to simulate this model. Generation of these paths requires the simulation of independent standard normal random variables at each time increment. Therefore, we need a good method to generate these standard normal random variables. We use two well known methods; Inverse transformation and the Box-Muller methods for generating the standard normal random variables. ${ }^{3}$

\section{The Monte Carlo Method for Option Pricing}

Consider the problem of estimating an integral over the unit interval. We can represent it in the following form ${ }^{1}$

$$
I=\int_{0}^{1} f(x) d x=\int_{0}^{1} f(x) p(x) d x
$$

where, $p$ is the uniform density. The basic Monte Carlo estimator is

$$
I_{N}=\frac{1}{N} \sum_{k=1}^{N} f\left(x_{k}\right)
$$

and the Monte Carlo error can be approximated by the sample standard deviation of ${ }^{5}$

$$
\sigma_{f}=E\left[f-E[f]^{2}\right]^{1 / 2}
$$

We assume that the underlying asset follows a Geometric Brownian motion where in a risk-neutral world, the drift term is equal to the risk free interest rate. In a continuous time notation, the stock price is:

$$
d S=r S d t+\sigma S d z
$$

Where $S$ is the stock price, $r$ is the risk-free interest rate, $\sigma$ is the volatility which we assumed to be a constant, and $d z$ is a Wiener process. Here, $\Delta z=\varepsilon \Delta t$ and $\varepsilon$ follows the standard normal distribution with mean zero and variance of one. Therefore, for a discrete time system, a change in stock price becomes

$$
\begin{aligned}
\Delta S & =r S \Delta t+\sigma S \Delta z \\
& =r S \Delta t+\sigma S \varepsilon \sqrt{\Delta t}
\end{aligned}
$$

Thus $\quad \frac{\Delta S}{S}=r \Delta t+\sigma \varepsilon \sqrt{\Delta t}$

which also follows a normal distribution, and represents a percentage change in stock return over a short time period $\Delta t$. Now we follow the procedure for Monte Carlo simulation and divide the lifespan of the stock into $n$ short 
intervals with length of $\Delta t$, then by applying Ito's Lemma to the stock price process, we get:

$$
\begin{aligned}
d \ln (S) & =\left(\frac{1}{S} r S+0+\frac{1}{2}\left(-\frac{1}{S^{2}}\right) \sigma^{2} S^{2}\right) d t+\frac{1}{S} \sigma S d z \\
& =\left(r-\frac{1}{2} \sigma^{2}\right) d t+\sigma d z
\end{aligned}
$$

By applying to discrete time notation, the above formula becomes:

$$
d \ln (S)=\left(r-\frac{1}{2} \sigma^{2}\right) \Delta t+\sigma \varepsilon \sqrt{\Delta t}
$$

Since $\Delta t$ represents short time interval, the change in stock price becomes:

$$
\begin{array}{r}
\ln \left(S_{t+\Delta t}\right)-\ln \left(S_{t}\right) \\
=\left(r-\frac{1}{2} \sigma^{2}\right) \Delta t+\sigma \varepsilon \sqrt{\Delta t}
\end{array}
$$

Which eventually gives the path generating formula for the stock price by applying Monte Carlo simulation

$$
S_{t+\Delta t}=S_{t} e^{\left(r-\frac{1}{2} \sigma^{2}\right) \Delta t+\sigma \varepsilon \sqrt{\Delta t}}
$$

Where, $S_{t}$ denotes the value of the stock at time $t, \varepsilon$ represents a number randomly sampled from a normal distribution with zero mean and standard deviation equals to one and when the volatility is constant, the parameters $\mu$ and $\sigma$ are also constant, which makes the above equation the true value of stocks, instead of an approximation following Geometric Brownian motion, which is then used to value the price of an European as well as various path dependent options by using Monte Carlo simulation. For this paper, the formula is encoded into MATLAB to create a series of random paths (Fig. 1) following Brownian motion. ${ }^{4}$

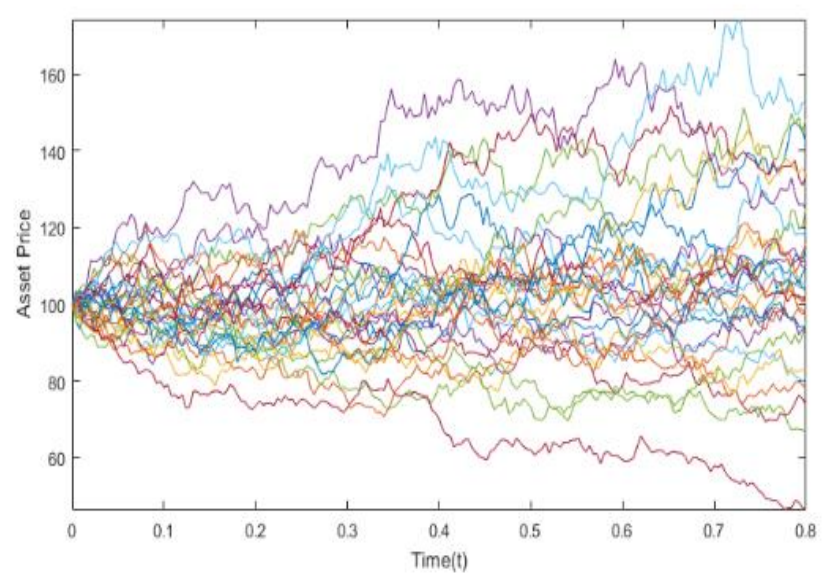

Fig. 1. Standard Construction of Brownian motion path

\section{Pseudorandom vs Quasi-Random Points}

The value of Monte Carlo integral highly depends on the choice of random number. The difference between quasiMonte Carlo and Monte Carlo is the way the random numbers are chosen. Quasi-Monte Carlo uses a lowdiscrepancy sequence such as the Halton sequence, the Sobol sequence, whereas Monte Carlo uses a pseudorandom sequence. The advantage of using lowdiscrepancy sequences is a faster rate of convergence. From Fig. 2, we see that Halton and Sobol sequence is more evenly distributed compared to Pseudorandom number.

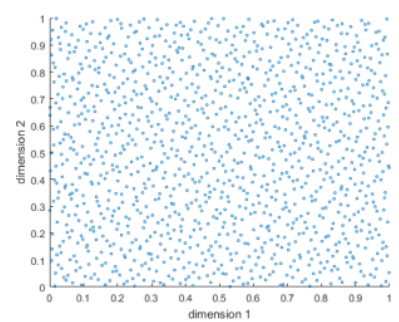

(a) Pseudorandom Numbers

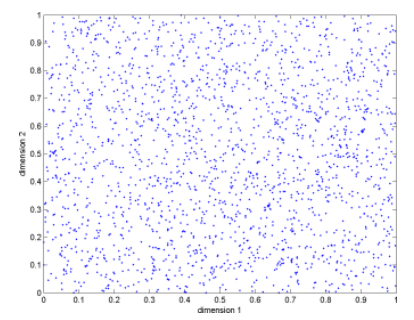

(b) Sobol sequence

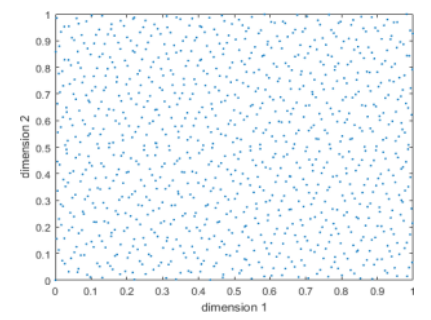

(c) Halton sequence

Fig. 2. Comparison: Random points vs Quasi-Random points (Sobol and Halton sequence).

\section{The Quasi-Monte Carlo Method}

Consider the following $d$ dimensional integral,

$$
\int_{[0,1)^{d}} f(x) d x
$$

The QMC method approximates this integral with

$$
\int_{[0,1) d} f(x) d x \approx \sum_{i=1}^{N} f\left(u_{i}\right)
$$

where, $u_{1}, \ldots, u_{N}$ are coming from a low discrepancy sequence instead of a pseudorandom numbers.

\section{MC and QMC Methods for European Call Option Pricing}

We consider an European Call options with initial stock price $S_{0}=90, \sigma=0.2 ; r=0.05 ; n=10,000$. By varying $K$ and $T$, we get Table 1 which shows that European call option price increases when time to maturity increases. For MC method, Antithetic variate gives better results than standard MC. For QMC method, both Halton and Sobol sequence gives the option price which is very close to $\mathrm{BS}$ option price.

Fig. 3(a) shows the European call option price with MC, AMC and QMC method by using Halton and Sobol sequence. From this figure, we see that QMC method converges much more faster than MC method. 
Table 1. European Call option pricing by MC and QMC Methods with $S_{0}=90, \sigma=0.2, r=0.05, n=10$, 000 for different $K$ and $T$.

\begin{tabular}{|c|l|r|c|c|c|c|}
\hline K & T & Exact & \multicolumn{2}{|c|}{ Monte Carlo } & \multicolumn{2}{c|}{ Quasi-Monte Carlo } \\
& & & Standard & AMC & Halton & Sobol \\
\hline \multirow{5}{*}{90} & 1 & 9.4055 & 9.1652 & 9.4655 & 9.4053 & 9.4048 \\
\cline { 2 - 7 } & 2 & 14.514 & 14.4594 & 14.5688 & 14.5138 & 14.5126 \\
\cline { 2 - 7 } & 3 & 18.8319 & 18.7706 & 18.7753 & 18.8317 & 18.8295 \\
\cline { 2 - 7 } & 4 & 22.6919 & 22.8713 & 22.9269 & 22.6920 & 22.6884 \\
\cline { 2 - 7 } & 5 & 26.2247 & 26.1451 & 26.1033 & 26.2250 & 26.2200 \\
\hline \multirow{5}{*}{100} & 1 & 5.0912 & 5.3314 & 5.2079 & 5.0917 & 5.0906 \\
\cline { 2 - 7 } & 2 & 9.9088 & 10.0278 & 9.9639 & 9.9091 & 9.9074 \\
\cline { 2 - 7 } & 3 & 14.1690 & 14.1451 & 14.2444 & 14.1698 & 14.1674 \\
\cline { 2 - 7 } & 4 & 18.0602 & 17.8771 & 18.0831 & 18.0606 & 18.0569 \\
\cline { 2 - 7 } & 5 & 21.6677 & 21.6358 & 21.5537 & 21.6682 & 21.6632 \\
\hline \multirow{4}{*}{110} & 1 & 2.5237 & 2.4919 & 2.5063 & 2.5243 & 2.5231 \\
\cline { 2 - 7 } & 2 & 6.5545 & 6.7856 & 6.5754 & 6.5554 & 6.5531 \\
\cline { 2 - 7 } & 3 & 10.4927 & 10.3730 & 10.6026 & 10.4937 & 10.4908 \\
\cline { 2 - 7 } & 4 & 14.2413 & 14.5854 & 14.2618 & 14.2423 & 14.2383 \\
\cline { 2 - 7 } & 5 & 17.7998 & 17.8581 & 17.8420 & 17.8008 & 17.7954 \\
\hline
\end{tabular}

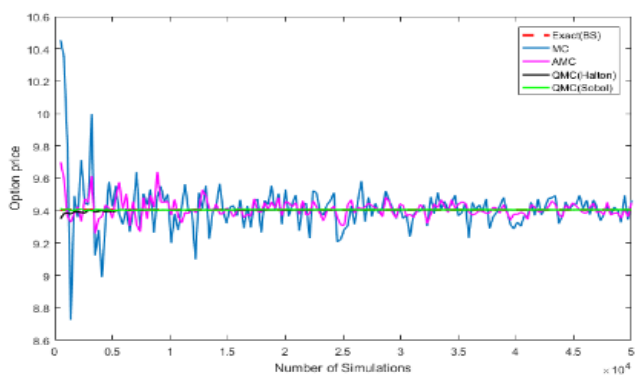

(a) Comparison of $\mathrm{MC}$ and $\mathrm{QMC}$ methods

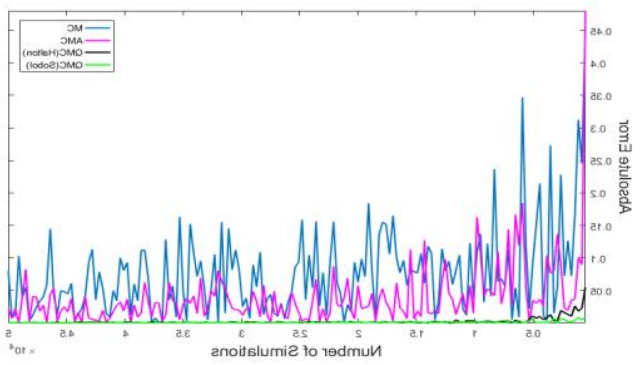

(b) Absolute error

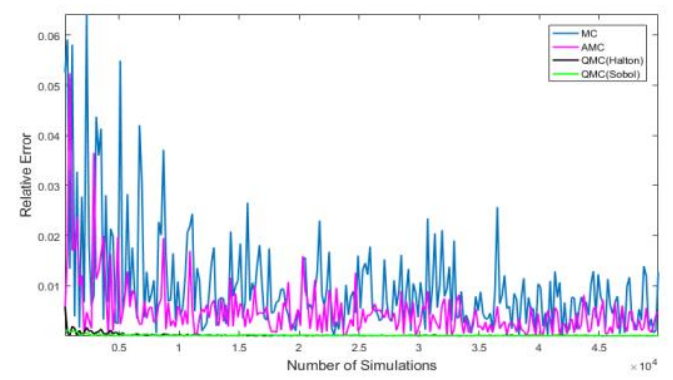

(c) Relative error

Fig. 3. Comparison and error of MC and QMC methods for European Call option price.

For higher number of simulations, both $\mathrm{MC}$ and QMC method converges to the BS option price. Fig. 3(b) shows the absolute error of MC and QMC method for European Call option pricing. As $n$ increases, the absolute error of both MC and QMC method decreases. Here, QMC method gives the lower relative error than MC method. From Fig. 3(c), we see that relative error of QMC method is very small compared with MC method.

\section{Comparison Between MC and QMC Methods for Asian Option Pricing}

The following Table 2 shows the arithmetic average Asian call option price with Standard MC, AMC and QMC method by using Halton and Sobol sequence by using the parameter $S_{0}=100 ; r=0.05 ; \sigma=0.2 ; T=1$ and by varying $K$ and $m$ (Number of Steps). Here, we consider extended BS formula for Asian option pricing as standard. ${ }^{2}$ When number of steps is higher, QMC method with Halton sequence gives worse result whereas QMC method with Sobol sequence gives better result for any number of steps.

Table 2. Asian option pricing by MC and QMC method with $S_{0}=100, r=0.05, \sigma=0.2, T=1$, $n=1,00,000$ for different $K$ and $m$.

\begin{tabular}{|c|c|c|c|c|c|c|}
\hline \multirow[t]{2}{*}{$K$} & \multirow[t]{2}{*}{$m$} & \multirow{2}{*}{$\begin{array}{l}\text { Exact } \\
\text { (BS) }\end{array}$} & \multicolumn{2}{|l|}{$\mathrm{MC}$} & \multicolumn{2}{|c|}{ QMC } \\
\hline & & & Standard & AMC & Halton & Sobol \\
\hline \multirow{6}{*}{90} & 10 & 12.6298 & 12.5835 & 12.5470 & 12.545 & 12.5393 \\
\hline & 20 & 12.6298 & 12.5766 & 12.5679 & 12.5684 & 12.5560 \\
\hline & 50 & 12.6298 & 12.5812 & 12.5982 & 14.211 & 12.5324 \\
\hline & 100 & 12.6298 & 12.6173 & \begin{tabular}{|l|}
12.5921 \\
\end{tabular} & 14.312 & 12.542 \\
\hline & 200 & 12.6298 & 12.5877 & 12.5986 & 14.0036 & 12.5534 \\
\hline & 500 & 12.6298 & 12.5920 & 12.5969 & 13.5334 & 12.5990 \\
\hline \multirow{6}{*}{100} & 10 & 5.7828 & 5.7038 & 5.6799 & 5.6628 & 5.6668 \\
\hline & 20 & 5.7828 & 5.7232 & 5.7347 & 5.7076 & 5.7073 \\
\hline & 50 & 5.7828 & 5.7464 & 5.7407 & 6.8855 & 5.7155 \\
\hline & 100 & 5.7828 & 5.7540 & 5.7625 & 6.9535 & 5.7192 \\
\hline & 200 & 5.7828 & 5.7630 & 5.7670 & 6.7308 & 5.7166 \\
\hline & 500 & 5.7828 & 5.7540 & 5.7594 & 6.3628 & 5.7493 \\
\hline \multirow{6}{*}{110} & 10 & 1.9711 & 1.9249 & 1.9262 & 1.9154 & 1.9215 \\
\hline & 20 & 1.9711 & 1.9310 & 1.9308 & 1.9523 & 1.9539 \\
\hline & 50 & 1.9711 & 1.9843 & 1.9794 & 2.5484 & 1.9542 \\
\hline & 100 & 1.9711 & 1.9649 & 1.9834 & 2.5660 & 1.9608 \\
\hline & 200 & 1.9723 & 1.9969 & \begin{tabular}{|l|}
1.9820 \\
\end{tabular} & 2.4454 & 1.9612 \\
\hline & 50 & 1.9114 & 1.9915 & 1.9932 & 2.2181 & 1.9769 \\
\hline
\end{tabular}

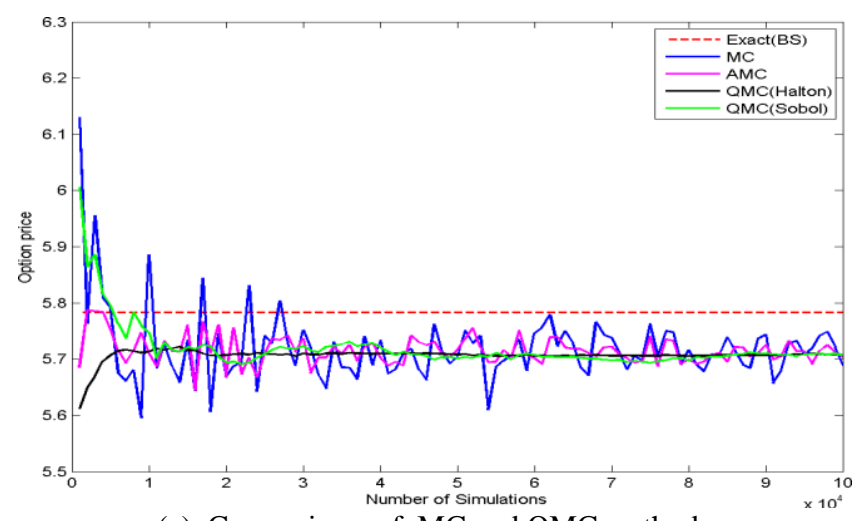

(a) Comparison of $\mathrm{MC}$ and QMC methods 


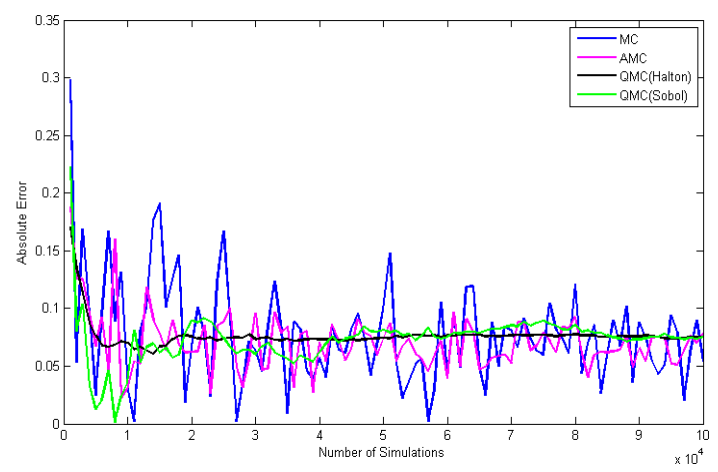

(b) Absolute error

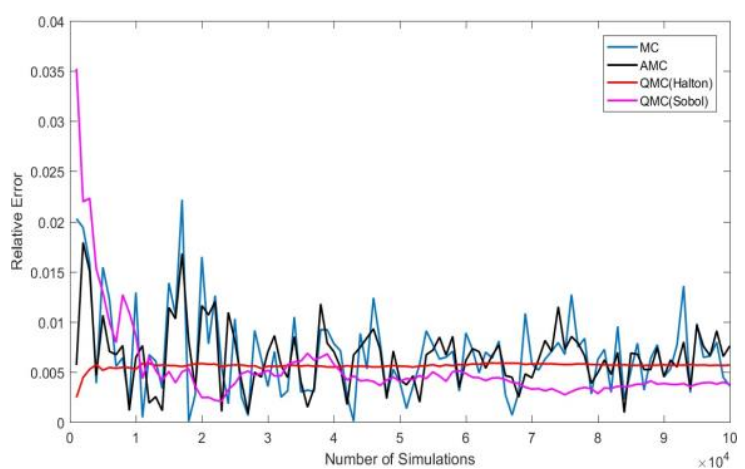

(c) Relative error

Fig. 4. Comparison and error of $\mathrm{MC}$ and QMC methods for arithmetic Asian call option price.

Fig. 4(a) shows the arithmetic average asian call option price by using MC, AMC and QMC methods. For higher number of simulation, both $\mathrm{MC}$ and QMC methods gives similar result but QMC method converges faster than MC method. Fig. 4(b) and 4(c) shows the absolute error and relative error of $\mathrm{MC}$ and QMC methods for arithmetic average Asian call option. Here, QMC methods gives lower absolute as well as relative error compared to $\mathrm{MC}$ methods.

\section{Barrier Option Pricing by MC and QMC Methods}

The following Table 3 shows the price of a Down-and-Out Barrier Call option with MC and QMC methods with $K=$ 100, $r=0.1, \sigma=0.3, T=0.2$ and by varying Initial Stock price $S_{0}$ and Barrier level $B$ by considering 100,000 random paths. Here, we consider extended $\mathrm{BS}$ formula for barrier option pricing as standard. ${ }^{7}$ From Table 3, we see that QMC methods (with Halton and Sobol sequence) gives better results compared to $\mathrm{MC}$ and $\mathrm{AMC}$ methods.

Fig. 5(a) shows the convergence of MC and QMC methods towards Extended BS for Down-and-Out Barrier Call option pricing. As number of simulations increases, both $\mathrm{MC}$ and QMC method gives option price which is very close to Extended BS price for Barrier option. The QMC method performed extremely well, as convergence is achieved faster and this was in connection to the use of Sobol sequences.
Table 3. Down and Out Barrier Call option pricing by MC and QMC methods with $K=100, r=0.1$, $\sigma=0.3, T=0.2$ and varying $S_{0}$ and $B$.

\begin{tabular}{|c|c|c|l|l|l|l|}
\hline$S_{0}$ & B & $\begin{array}{c}\text { Exact } \\
(B S)\end{array}$ & MC & \multicolumn{2}{c|}{ QMC } \\
& & Standard & AMC & Halton & Sobol \\
\hline 100 & 80 & 6.3425 & 6.3524 & 6.3325 & 6.3420 & 6.3232 \\
\cline { 2 - 7 } & 85 & 6.3076 & 6.3364 & 6.3169 & 6.3273 & 6.3084 \\
\cline { 2 - 7 } & 90 & 5.9772 & 6.1583 & 6.1399 & 6.1485 & 6.1339 \\
\cline { 2 - 7 } & 95 & 4.3975 & 5.1613 & 5.1411 & 5.1373 & 5.1206 \\
\hline 105 & 80 & 9.6158 & 9.6275 & 9.5974 & 9.6104 & 9.5854 \\
\cline { 2 - 7 } & 85 & 9.6052 & 9.6234 & 9.5934 & 9.6060 & 9.5808 \\
\cline { 2 - 7 } & 90 & 9.4733 & 9.5581 & 9.5283 & 9.5423 & 9.5176 \\
\cline { 2 - 7 } & 95 & 8.6732 & 9.0875 & 9.0603 & 9.0681 & 9.0445 \\
\hline 110 & 80 & 13.484 & 13.5006 & 13.4616 & 13.475 & 13.4442 \\
\cline { 2 - 7 } & 85 & 13.481 & 13.4996 & 13.4603 & 13.473 & 13.4433 \\
\cline { 2 - 7 } & 90 & 13.431 & 13.4768 & 13.4387 & 13.452 & 13.4185 \\
\cline { 2 - 7 } & 95 & 13.051 & 13.2736 & 13.2315 & 13.247 & 13.2195 \\
\hline 115 & 80 & 17.793 & 17.8113 & 17.7680 & 17.787 & 17.7434 \\
\cline { 2 - 7 } & 85 & 17.792 & 17.8110 & 17.7677 & 17.787 & 17.7431 \\
\cline { 2 - 7 } & 90 & 17.774 & 17.8031 & 17.7599 & 17.778 & 17.7356 \\
\cline { 2 - 7 } & 95 & 17.604 & 17.715 & 17.6739 & 17.695 & 17.6451 \\
\hline
\end{tabular}

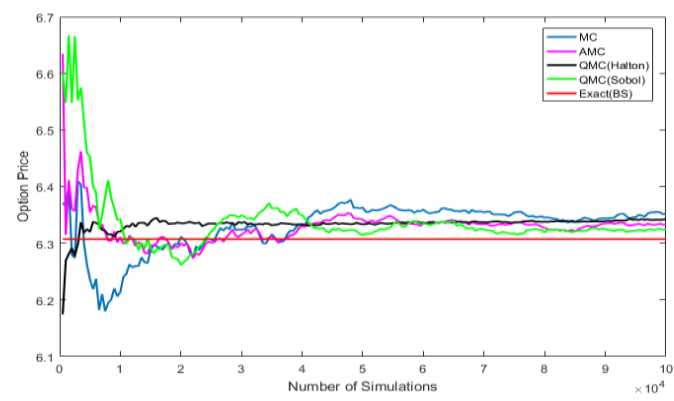

(a) Comparison of MC and QMC methods

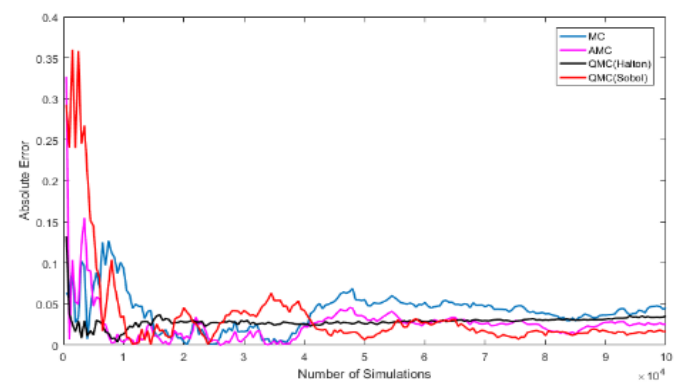

(b) Absolute error of MC and QMC methods

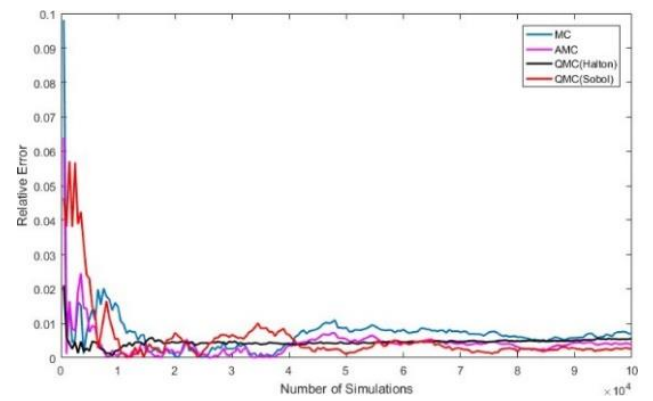

(c) Relative error of MC and QMC methods

Fig. 5. Comparison, absolute and relative error of $\mathrm{MC}$ and QMC methods for Barrier option price. 
Fig. 5(a) shows the comparison between MC and QMC methods along with the Exact result obtained by using Black Scholes Option Pricing formula. Fig. 5(b) and 5(c) shows the absolute and relative error of MC and QMC methods for a Down-and-Out Barrier Call option pricing. The relative errors from both simulations are reducing with an increase in the number of simulations. The relative errors obtained using the QMC method are generally lesser when compared to that of MC method.

\section{Comparison Between MC and QMC Methods for American Put Option Pricing}

We consider a non dividend American put option with $r=0.04 ; \sigma=0.2 ; T=1 ; n=10,000$. By varying $S_{0}$ and $K$, we get Table 4 by using MC and QMC methods by considering Halton and Sobol sequence.

Since we do not know the analytical solution of American option pricing, we compare the standard error $\left(\frac{\sigma}{\sqrt{n}}\right)$ of MC and QMC methods which is the statistical convergence rate of the above mentioned method. From the following Table 4, we observe that for higher Strike Prices, both MC and QMC methods gives very similar price for American put Option. On the other hand, for smaller strike price, we observe some fluctuations between $\mathrm{MC}$ and $\mathrm{QMC}$ methods.

Table 4. MC and QMC methods for American Put Option Pricing with $r=0.04, \sigma=0.2, T=1$ for different $S_{0}$ and $K$.

\begin{tabular}{|c|l|l|l|l|}
\hline$S 0$ & \multicolumn{1}{|c|}{$K$} & \multicolumn{1}{|c|}{$\begin{array}{c}\text { MC } \\
\text { (Standard) }\end{array}$} & $\begin{array}{c}\text { QMC } \\
\text { (Halton) }\end{array}$ & $\begin{array}{c}\text { QMC } \\
(\text { Sobol })\end{array}$ \\
\hline \multirow{4}{*}{80} & 80 & 5.0521 & 4.4638 & 5.1541 \\
\cline { 2 - 5 } & 90 & 11.2444 & 10.8276 & 11.2763 \\
\cline { 2 - 5 } & 100 & 19.9902 & 19.9334 & 19.9550 \\
\cline { 2 - 5 } & 110 & 29.9208 & 29.9126 & 29.9075 \\
\hline \multirow{4}{*}{85} & 80 & 3.3029 & 2.7160 & 3.3904 \\
\cline { 2 - 5 } & 90 & 8.0972 & 7.5011 & 8.2286 \\
\cline { 2 - 5 } & 100 & 15.5445 & 15.2388 & 15.4631 \\
\cline { 2 - 5 } & 110 & 24.9487 & 24.9114 & 24.9043 \\
\hline \multirow{4}{*}{90} & 80 & 2.0735 & 1.6210 & 2.1534 \\
\cline { 2 - 5 } & 90 & 5.6836 & 5.0218 & 5.7984 \\
\cline { 2 - 5 } & 100 & 11.7457 & 11.2139 & 11.7958 \\
\cline { 2 - 5 } & 110 & 20.1291 & 19.9336 & 20.0162 \\
\hline \multirow{3}{*}{95} & 80 & 1.2536 & 0.9415 & 1.3218 \\
\cline { 2 - 5 } & 90 & 3.8982 & 3.2332 & 4.0062 \\
\cline { 2 - 5 } & 100 & 8.6792 & 8.0148 & 8.8226 \\
\cline { 2 - 5 } & 110 & 15.8798 & 15.4734 & 15.8449 \\
\hline
\end{tabular}

Fig. 6(a) shows the price of a non-dividend american put options with $\mathrm{MC}$ (pseudorandom number) and QMC (Halton and Sobol sequence) methods. ${ }^{6}$

As the number of simulations increases, the fluctuation of $\mathrm{MC}$ method decreases. For higher number of simulations, both MC and QMC gives the similar results. Fig. 6(b) shows the standard error $\left(\frac{\sigma}{\sqrt{n}}\right)$ of MC and QMC methods for
American put option pricing where $\sigma$ is the standard deviation and $n$ is the number of Simulations. For large number of simulations, the standard error becomes very small for both MC and QMC methods.

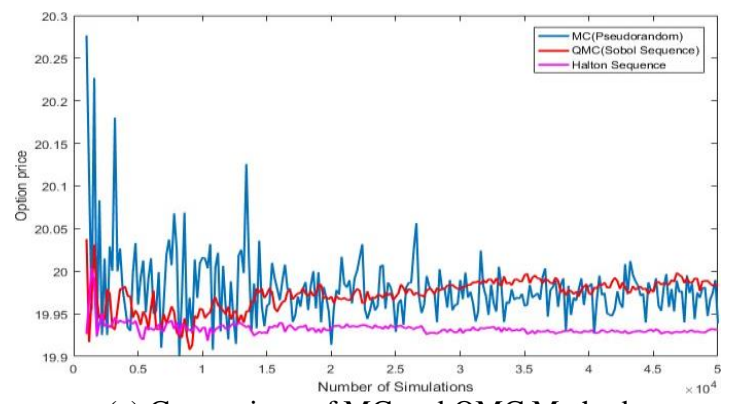

(a) Comparison of MC and QMC Method

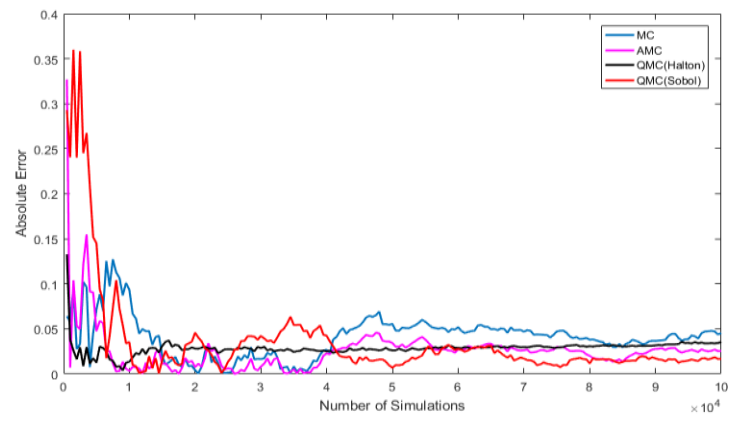

(b) Standard error of MC and QMC Methods

Fig. 6. Comparison and Standard error of MC and QMC methods for American option pricing.

\section{Conclusion}

In many computational finance problems, asset prices are modeled using the geometric Brownian motion. The simulation of the geometric Brownian motion involves generation of uniform random numbers, sampling from the normal distribution and construction of the Brownian motion paths. We can easily estimate the price of European Options by BS Model. But, for pricing strong path dependent options, there is no other choice but to use Monte Carlo Simulation. We observe that the ordinary MC method underpriced the option in most of the cases, as can be seen in the high discrepancies of the simulated values in comparison to the exact prices. It was also observed that using the AMC method resulted in the reduction of variance estimate when compared to the independent simulations obtained from the standard MC method. This was made possible since the antithetic variables used are negatively correlated and thus, increasing one variable led to a simultaneous decrease in the other variable. Hence, the AMC method gave out a better and fairly accurate result when compared to the MC method. Furthermore, the rate of convergence is accelerated when the simulation nodes are chosen deterministically as observed by the use of low discrepancy sequences. Thus, the use of QMC method together with Halton and Sobol sequences resulted to a more efficient method for valuing complex derivative like Asian, Barrier and American options. 
The Monte Carlo convergence rate is $O\left(N^{-1 / 2}\right)$ whereas the quasi-Monte Carlo convergence rate is $O\left((\log N)^{d} / N\right)$, where $N$ is sample size and $d$ is the dimension of the problem.

Finally, we can conclude that QMC techniques improve the efficiency in most of the cases because standard deviations were always lower for QMC than MC methods. For most of the cases, QMC has also the superior convergence rate compared to MC method.

\section{References}

1. Glasserman, P. 2003. Monte Carlo Methods in Financial Engineering. Springer-Verlag, York.

2. Privault, N., 2013. Stochastic Finance: An Introduction with Market Examples. CRC Press.

3. John C. H., 2015-16. Option, Futures and Other Derivatives, Global edition, Eighth edition, Pearson Education Limited.
4. Higham, D. J, 2013. An introduction to financial option valuation: mathematics, stochastics and computation. 13. Cambridge University Press.

5. Goncu, A, 2013. Monte Carlo and quasi-Monte Carlo methods in financial derivative pricing (Doctoral dissertation, The Florida State University). Chicago.

6. Longstaff, F. A., E. S, Schwartz, 2001. Valuing American Options by Simulation: a Simple Least-Squares Approach. Review of Financial Studies.

7. Broadie, M. P. Glasserman, and S.G. Kou, 2013. A continuity correction for discrete barrier options. Mathematical Finance, 7(4), 325-349.

8. Corwin Joy, Phelim P. Boyle, and Ken Seng Tan, 1996. Quasi-Monte Carlo Methods in Numerical Finance. Manage. Sci. 42, 6 (June 1996), 926-938 